\title{
Multidetector computed tomography for preoperative identification of left gastric vein location in patients with gastric cancer
}

\author{
Kentaro Kawasaki, Shingo Kanaji, Iwao Kobayashi, Tadatoshi Fujita, Hiroaki Kominami, Kiminiko Ueno, \\ Shinobu Tsutida, Masakazu Ohno, Masato Ohsawa, Yasuhiro Fujino, Masahiro Tominaga, \\ and TAKESHI NAKAMURA
}

Division of Gastroenterological Surgery, Hyogo Cancer Center, 13-70 Kitaouji-cho, Akashi, Hyogo 673-8558, Japan

\begin{abstract}
Background. The area near the left gastric vein (LGV) is a challenging site at which to perform dissection of the lymph nodes during gastrectomy. Therefore, knowledge of the precise location of the LGV is important. The objective of this study was to examine the usefulness of multidetector computed tomography (MDCT) for the identification of the LGV.

Methods. Eighty-one patients with gastric cancer underwent MDCT, which was performed with contrast media in $\mathbf{7 6}$ patients and without contrast media in 5 patients. A $5-\mathrm{mm}$ thin slice of the frontal image was reconstructed. These images were examined preoperatively to detect the location of the LGV. Upon gastrectomy, the LGV was identified and its location compared to that determined by MDCT.

Results. The LGV was identified by MDCT in 76 of the 81 patients $(93.8 \%)$. The LGV was subsequently located during the operation in all 81 patients. The LGV was located dorsal to the common hepatic artery in 40 patients $(\mathbf{4 9 . 4} \%)$, ventral to the common hepatic artery in 18 patients $(22.2 \%)$, ventral to the splenic artery in 17 patients $(21.0 \%)$, dorsal to the splenic artery in 2 patients $(2.5 \%)$ and in other positions in 4 patients $(4.9 \%)$. In all patients, the location of the LGV detected using MDCT was consistent with that identified during gastrectomy. In the 4 patients with relatively unusual locations of the LGV, these $4 \mathrm{LGV}$ variants were identified preoperatively by MDCT.

Conclusion. MDCT was useful for identifying the location of the LGV prior to gastrectomy.
\end{abstract}

Key words Left gastric vein · Multidetector computed tomography $\cdot \mathrm{MDCT} \cdot$ Gastric cancer

\section{Introduction}

In Japan, the perigastric lymph nodes are usually dissected in patients with gastric cancer $[1,2]$. In our expe-

Offprint requests to: $\mathrm{K}$. Kawasaki

Received: July 8, 2009 / Accepted: November 3, 2009 rience, the area near the left gastric vein (LGV) is one of the most challenging sites at which to perform dissection, because of its various anatomical variants. Therefore, knowledge of the precise location of the LGV preoperatively may make it possible to avoid unnecessary bleeding.

In Japan, computed tomography (CT) scanning from the chest to the abdomen is routinely performed to check for the presence of metastases in patients with gastric cancer. Three-dimensional computed tomographic angiography (3DCTA) is a reliable method for determining the location and anatomy of the vessels around the stomach [3-8]. However, this method requires an additional $\mathrm{CT}$ scan over and above the routine $\mathrm{CT}$, so extra cost is incurred. On the other hand, the recent development of multidetector computed tomography (MDCT) now makes it possible to obtain highly detailed information, including high-resolution data on perigastric vascular anatomy.

In the present study, the location of the LGV was assessed during preoperative MDCT used to perform the routine check for metastasis, and these preoperative results were compared to the location of the LGV visualized during the actual operation. The aim of this study was to examine the usefulness of MDCT for identification of the location of the LGV.

\section{Patients and methods}

\section{Patients}

Eighty-one patients with primary gastric cancer were enrolled in this study. All patients underwent MDCT preoperatively to detect metastasis from the chest to the abdomen. MDCT was performed with contrast media in 76 patients and without contrast media in 5 patients who were allergic to contrast agents. In one patient with arterial and venous variants detected by MDCT, 

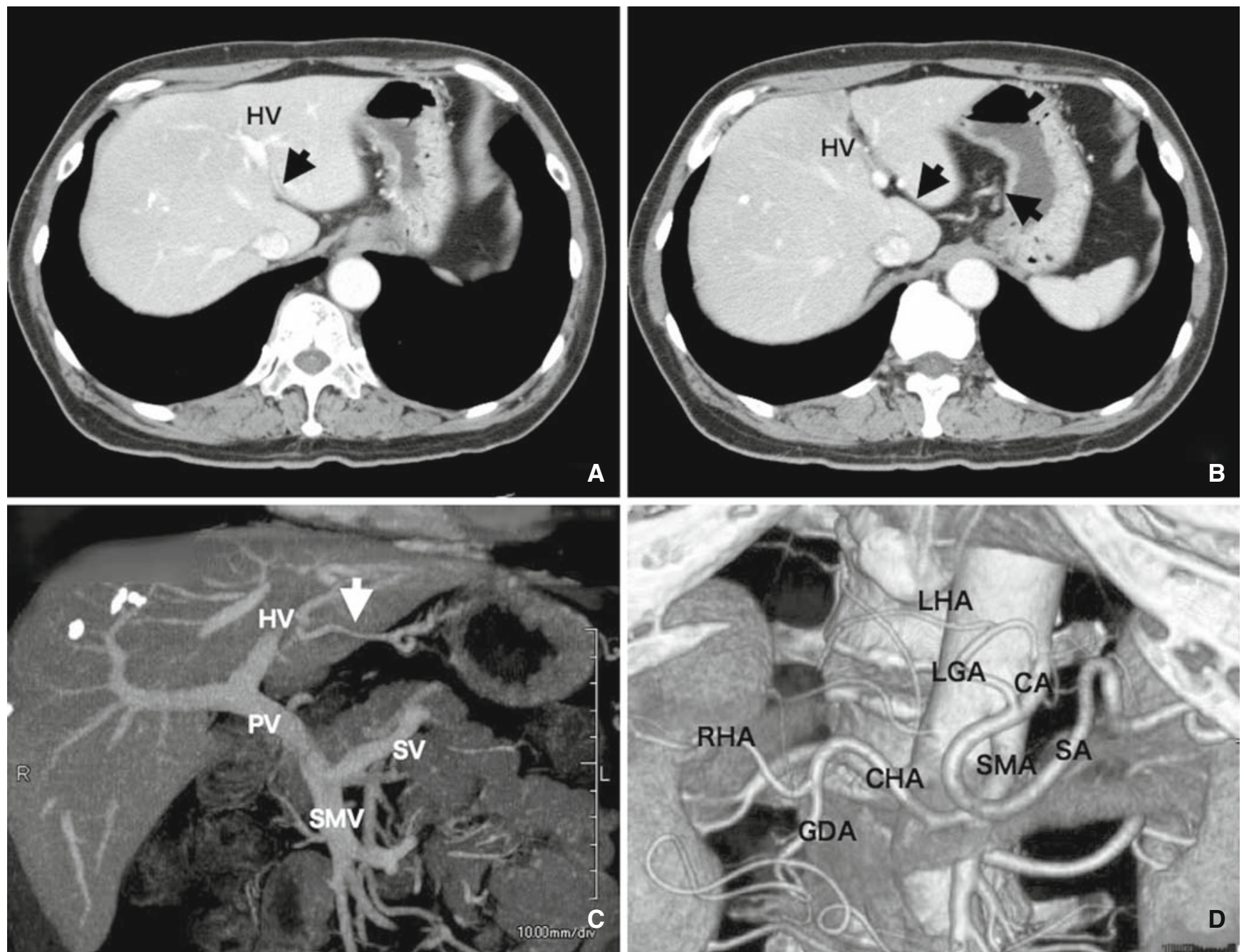

Fig. 1A-D. Multidetector computed tomography (MDCT) and three-dimensional computed tomographic angiography (3DCTA) imaging in case 31; arterial and venous variants were detected by MDCT (A and B), so 3DCTA was performed additionally ( $\mathbf{C}$ and $\mathbf{D})$. The left gastric vein (LGV; arrow) starts from the stomach $(\mathbf{B})$ and ends in the hepatic vein $(H V ; \mathbf{A})$. The venous phase of the 3DCTA imaging showed that the LGV flowed into the HV (C). The arterial

3DCTA was performed additionally (Fig.1). All patients underwent gastrectomy, performed at the Gastroenterological Department of Surgery, Hyogo Cancer Center, Hyogo, Japan, between September 2007 and May 2009. Open gastrectomy was performed in 34 patients and laparoscopic gastrectomy in 47 patients.

\section{MDCT and location of $L G V$}

MDCT was performed using an Aquilion 16-row multidetector CT scanner (Toshiba Medical Systems, Tokyo, Japan). Contrast medium (100 $\mathrm{ml}$ at a rate of $2 \mathrm{ml} / \mathrm{s}$ ) was intravenously injected. After $70 \mathrm{~s}$, the patients were scanned from the chest to abdomen. A

phase of the 3DCTA imaging showed that the common hepatic artery $(C H A)$ started from the superior mesenteric artery (SMA), and the left hepatic artery ( $L H A)$ started from the left gastric artery $(L G A ; \mathbf{D}) . S A$, Splenic artery; $C A$, celiac artery; $P V$, portal vein; $S V$, splenic vein; $S M V$, superior mesenteric vein; $R H A$, right hepatic artery; $G D A$, gastroduodenal artery; $H V$, hepatic vein

5-mm thin slice of the standard frontal image was reconstructed, and these images were examined preoperatively by surgeons (K. K. and S. K.) to detect the location of the LGV. For the purposes of this study, the vessel connecting the portal or splenic vein to the stomach was defined as the LGV (Figs. 2 and 3). The LGV was described as one of the following five types based on its location: (A) dorsal to the common hepatic artery, (B) ventral to the common hepatic artery, (C) ventral to the splenic artery, (D) dorsal to the splenic artery, or (E) other (Fig. 4). The location of the LGV was confirmed during the gastrectomy, and this result was compared to the location determined preoperatively by MDCT imaging. 

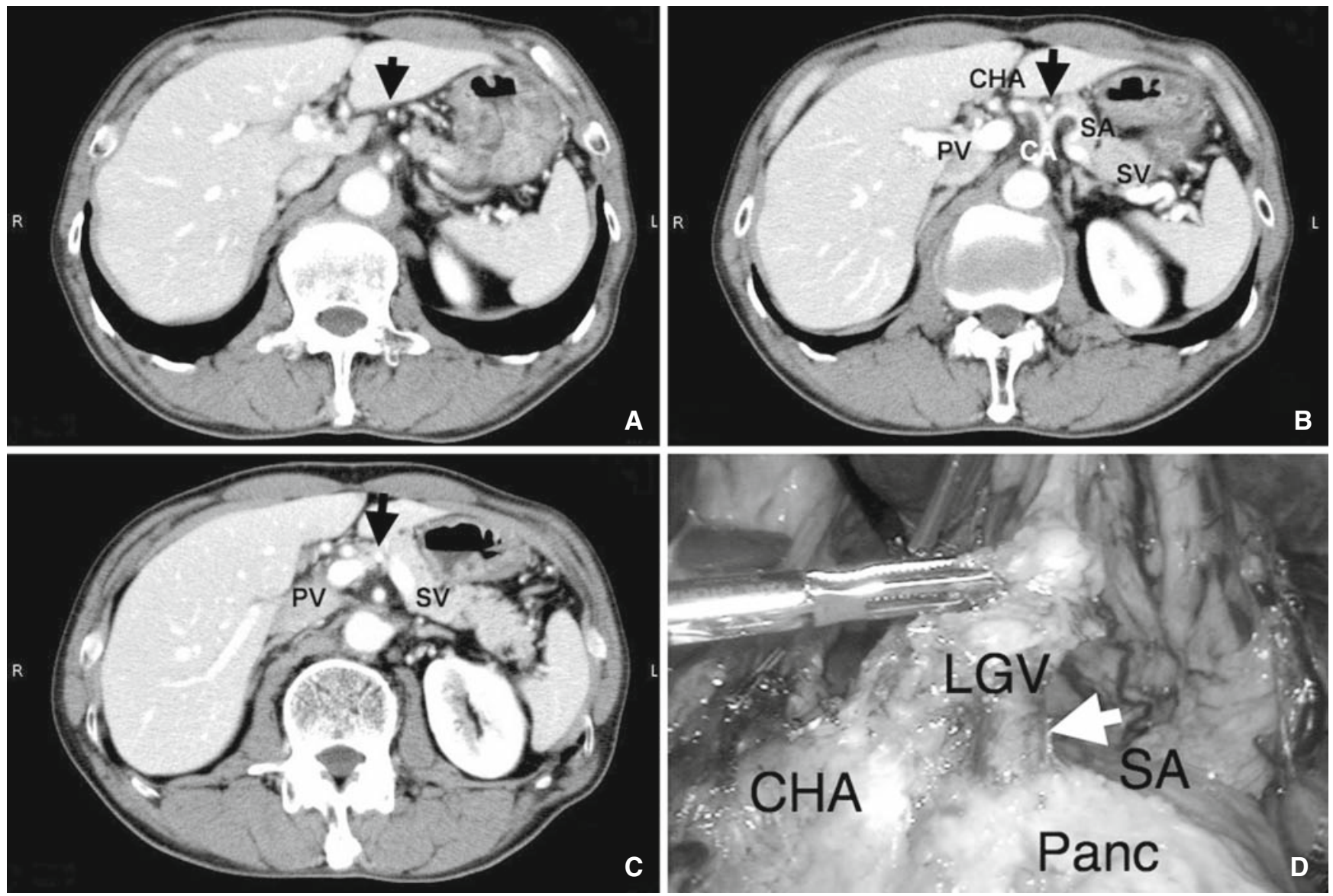

Fig. 2A-D. Multidetector computed tomography (MDCT) imaging with contrast medium in case 35: the LGV (arrow) starts from the stomach $(\mathbf{A})$, runs between the CHA and SA $(\mathbf{B})$, and ends in the SV $(\mathbf{C})$. Upon laparoscopic gastrectomy, the location of the LGV was confirmed (D). Panc, Pancreas

\section{Results}

\section{Identification of $L G V$}

The LGV was identified by MDCT preoperatively in 76 of the 81 patients $(93.8 \%)$, including 3 of the 5 patients scanned without contrast media.

\section{Location of the LGV identified during gastrectomy}

The location of the LGV was found to be type A in 40 patients $(49.4 \%)$, type $\mathrm{B}$ in $18(22.2 \%)$, type $\mathrm{C}$ in 17 $(21.0 \%)$, type D in $2(2.5 \%)$, and type $\mathrm{E}$ in $4(4.9 \%$; Table 1). In the 4 type $\mathrm{E}$ patients, the LGV flowed into the hepatic vein in 2 patients, and the LGV was absent and a big right gastric vein was confirmed in 2 patients.

\section{Comparison of $L G V$ locations identified by MDCT versus surgery}

The location of the LGV detected by preoperative MDCT examination was consistent with that identified
Table 1. Location of the LGV

\begin{tabular}{lcc}
\hline Type & MDCT & Operation (\%) \\
\hline A & 37 & $40(49.4)$ \\
B & 18 & $18(22.2)$ \\
C & 15 & $17(21.0)$ \\
D & 2 & $2(2.5)$ \\
E & 4 & $4(4.9)$ \\
Unknown & 5 & $0(0)$ \\
Total & 81 & 81 \\
Accuracy & $93.8 \%(76 / 81)$ & \\
\hline
\end{tabular}

LGV, left gastric vein; MDCT, multidetector computed tomography

during gastrectomy in $100 \%$ of the 76 cases of successful detection by MDCT (Table 1). The 4 type E cases of LGV variants were all identified preoperatively by MDCT.

\section{Other findings}

In 3 of the 81 patients, the common hepatic artery was absent, and this vascular variant was detected by MDCT before the operation. 

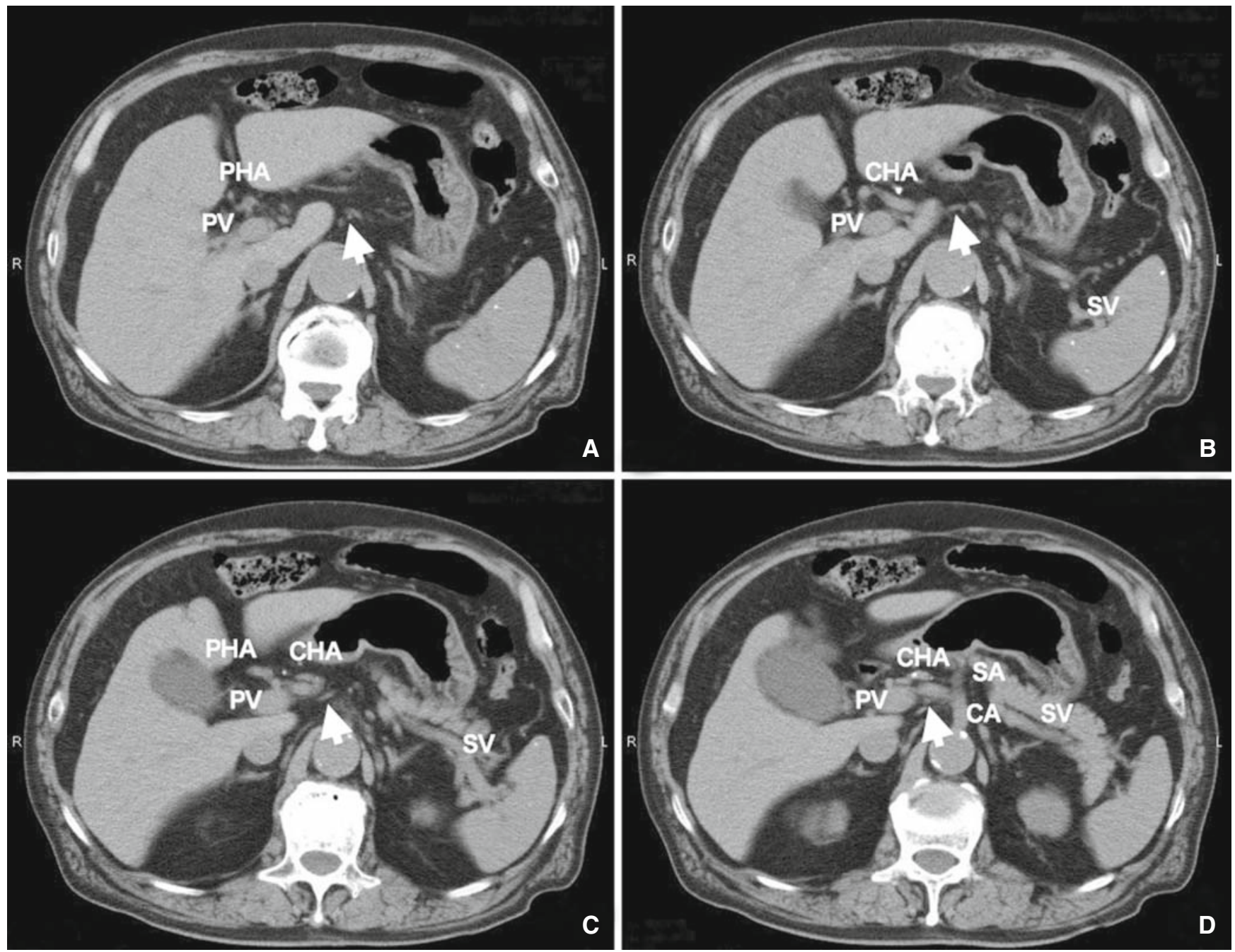

Fig. 3A-D. MDCT imaging without contrast medium in case 55: the LGV (arrow) starts from the stomach (A, B, and $\mathbf{C})$, runs dorsal to the CHA (C), and ends in the PV (D). PHA, Proper hepatic artery

\section{Discussion}

The technique of 3DCTA is reported to be useful for visualizing the anatomy of the vessels around the stomach [3-8]. However, 3DCTA is expensive, and it requires an additional $\mathrm{CT}$ scan using a substantial amount of contrast medium. On the other hand, the recently developed MDCT technique is able to generate high-resolution images of perigastric vessels. In addition, these MDCT images can be interpreted on a computer monitor by displaying scan results stored in the patient's electronic medical records. The anteroposterior relationship of the MDCT images is easily recognized. Among the perigastric vessels, the LGV is easy to detect, because it starts directly from the stomach. Moreover, as the LGV is one of several vessels to be ligated during lymph node dissection above the pancreas, identifying its location is particularly useful.
Therefore, MDCT proved valuable in preoperative visualization of the LGV in the present study.

In general, anatomical examination around the LGV clarifies the relationship between the LGV and the portal or splenic vein $[9,10]$. However, the portal or splenic vein is sometimes difficult to locate during laparoscopic gastrectomy, so the common hepatic artery and the splenic artery were used as the landmarks in the present study.

In 76 of the 81 patients in this study, the LGV could be detected by MDCT preoperatively. The accuracy of detecting the correct location of the LGV by preoperative MDCT (relative to the location determined during gastrectomy) was $93.8 \%$ in this study. Moreover, the LGV was detected in 3 of the 5 patients in whom contrast media could not be used. Therefore, the preoperative information provided by MDCT is extremely reliable and useful for planning surgical strategies. 


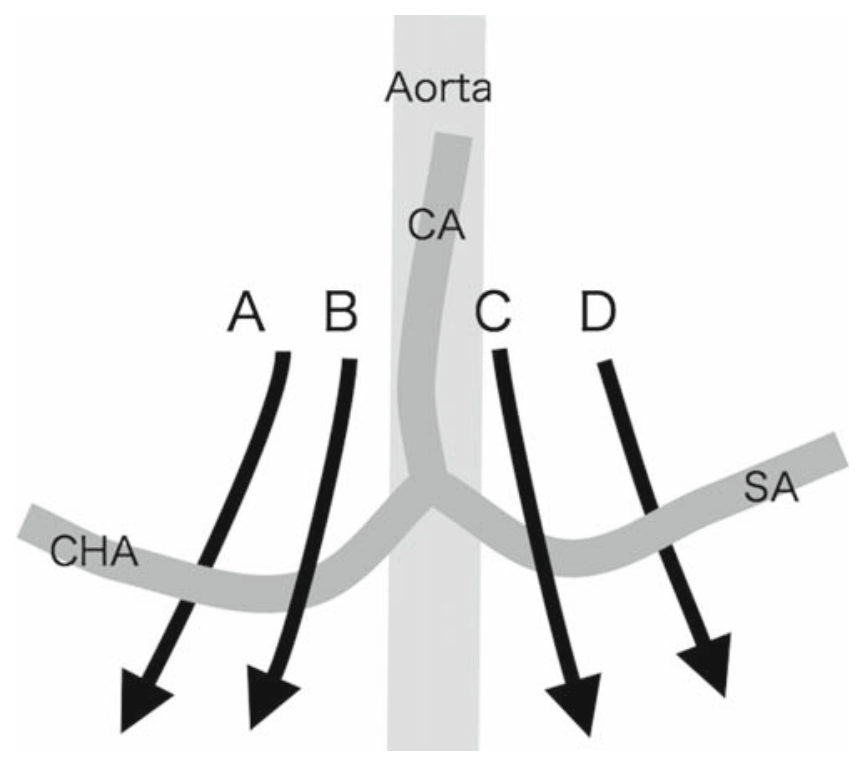

Fig. 4. The location of the LGV was described as one of five types: $A$ (dorsal to the common hepatic artery), $B$ (ventral to the common hepatic artery), $C$ (ventral to the splenic artery), $D$ (dorsal to the splenic artery), and E (other; not shown)

In the three patients in whom the LGV was not detected preoperatively using MDCT with contrast media, the preoperative MDCT image was re-examined postoperatively. However, the LGV was not visible on the MDCT image, even postoperatively when the location of the LGV was known based on surgical findings. It was determined that, in all three patients, there was no vessel connecting to the stomach. These three patients had advanced gastric cancer with lymph node metastasis above the pancreas; therefore, the blood flow though the LGV might not have been adequate to allow detection. However, this difficulty in identifying the LGV might be also attributed to the 5-mm slice thickness of the MDCT scan image. Therefore, in patients in whom the LGV cannot be found by MDCT, the use of 3DCTA is recommended.

In the four patients with type E ("other") location, the LGV was not found at the usual anatomical site. The LGV flowed into the hepatic vein in two patients, and the LGV was absent and the right gastric vein was confirmed in two patients. Although the anatomy of the perigastric arteries was not examined in the present study, these arteries can be visualized by MDCT in most cases. In three patients, the common hepatic artery was found to be absent preoperatively. Such information is useful to surgeons, especially for the preoperative planning of surgical strategy.

This study investigated the usefulness of MDCT for the identification of the LGV before gastrectomy. It is clear that the higher resolution of newly developed MDCT imaging provides highly detailed and accurate information. The interpretation of the results from MDCT is easily performed on-screen using the electronically stored images. We recommend that surgeons examine MDCT images preoperatively in order to plan their surgical strategy, thereby optimizing the safety and efficacy of gastrectomy, especially laparoscopic gastrectomy.

\section{References}

1. Shiraishi N, Yasuda K, Kitano S. Laparoscopic gastrectomy with lymph node dissection for gastric cancer. Gastric Cancer 2006; 9:167-76.

2. Kitano S, Shiraishi N, Fujii K, Yasuda K, Inomata M, Adachi Y. A randomized controlled trial comparing open vs laparoscopyassisted distal gastrectomy for the treatment of early gastric cancer: an interim report. Surgery 2002;131:306-11.

3. Takiguchi S, Sekimoto M, Fujiwara Y, Yasuda T, Yano M, Hori $\mathrm{M}$, et al. Laparoscopic lymph node dissection for gastric cancer with intraoperative navigation using three-dimensional angio computed tomography images reconstructed as laparoscopic view. Surg Endosc 2004;18:106-10.

4. Kim HS, Han HY, Choi JA, Park CM, Cha IH, Chung KB, et al. Preoperative evaluation of gastric cancer: value of spiral CT during gastric arteriography (CTGA). Abdom Imaging 2001;26: 123-30.

5. Kumano S, Tsuda T, Tanaka H, Hirata M, Kim T, Murakami T, et al. Preoperative evaluation of perigastric vascular anatomy by three-dimensional computed tomographic angiography using 16channel multidetector-row computed tomography for laparoscopic gastrectomy in patients with early gastric cancer. J Comput Assist Tomogr 2007;31:93-7.

6. Matsuki M, Kani H, Tatsugami F, Yoshikawa S, Narabayashi I, Lee SW, et al. Preoperative assessment of vascular anatomy around the stomach by 3D imaging using MDCT before laparoscopy-assisted gastrectomy. AJR Am J Roentgenol 2004; 183:145-51.

7. Matsumoto A, Kitamoto M, Imamura M, Nakanishi T, Ono C, Ito $\mathrm{K}$, et al. Three-dimensional portography using multislice helical CT is clinically useful for management of gastric fundic varices. AJR Am J Roentgenol 2001;176:899-905.

8. Matsuki M, Tanikake M, Kani H, Tatsugami F, Kanazawa S, Kanamoto T, et al. Dual-phase 3D CT angiography during a single breath-hold using 16-MDCT: assessment of vascular anatomy before laparoscopic gastrectomy. AJR Am J Roentgenol 2006;186:1079-85.

9. Roi DJ. Ultrasound anatomy of the left gastric vein. Clin Radiol 1993;47:396-8.

10. Ibukuro K, Tsukiyama T, Mori K, Inoue Y. Peripancreatic veins on thin-section (3 mm) helical CT. AJR Am J Roentgenol 1996; 167:1003-8. 